by $70^{\circ}$. Additional polarized radiation must occur between the subpulses to prevent the polarization dropping below 50 per cent. The rate of change of direction of polarization for the radiation between the subpulses agrees with the simple model. The rate across the subpulses is considerably lower than expected, however; it could be due to the presence of discrete regions of radiation across which the polarization is relatively constant. The symmetry shown in the variation of polarization suggests that these regions are part of a symmetrical feature such as a conical annulus of emission centred on the magnetic pole. The subpulses would occur when the annulus passes through the line of sight, while the weaker radiation between the subpulses would come from near the pole.

To summarize, the results at $4,830 \mathrm{MHz}$ are generally in agreement with an oblique rotator model for a pulsar, although a more complex radiation distribution is required than at lower frequencies. The large variations in pulse shape and intensity seem to arise in the mechanism producing the radiation, for the increase of variability with frequency rules out scintillation in the intervening medium. The mean energy spectrum points to a radiation cut-off frequency near $5 \mathrm{GHz}$.

The investigation was suggested to us by J. G. Bolton. We thank D. J. Cooke for setting up the equipment for the observations. The NRAO parametric amplifier was on loan by courtesy of Dr D. S. Heeschen.

F. F. GarDner

CSIRO Radiophysies Laboratory,

J. B. WHITEOAK

Sydney, Australia.

Received August 18, 1969 .

${ }^{2}$ Radhakrishnan, V., Cooke, D. J., Komesaroff, M. M., and Morris, D., Nature, 221, $443(1969)$.

'Radhakrishnan, V., and Cooke, D. J., Astrophys. Lett., 3, 225 (1969).

'Wielebinski, R., Proc. Astron. Sor. Austral., 1, 226 (1969).

\section{Absorption of High Energy Gamma-rays in the Vicinity of Pulsar NP 0532}

THE purpose of this article is to point out that photonphoton absorption may remove high energy $\gamma$-rays from the vicinity of pulsar NP 0532 in the Crab Nebula. The pulsar was discovered at radio frequencies by Staelin and Reifenstein $^{1}$, and recently Cocke, Disney and Taylor ${ }^{2}$ discovered strong optical pulses. Fritz et al. ${ }^{3}$ have found pulsed X-rays. The pulse durations at the three different frequencies are similar.

In recent years the effects of photon-photon absorption have been pointed out by many authors ${ }^{4-6}$. In addition, Jelley $^{2}$ has considered the same absorption process within the possible sources of $\gamma$-rays. The absorption of a $\gamma$-ray of energy $E_{\gamma}$ by a photon of energy $\varepsilon$ has a threshold at $E_{\gamma} \varepsilon(1-\cos \theta)=2\left(m c^{2}\right)^{2}$, where $m c^{2}$ is the rest energy of the electron and $\theta$ the angle between their directions of motion. The cross-section $\sigma$ for the process rises from zero at threshold to a maximum just above it. For an isotropic distribution of photons we take $\sigma$ to be $10^{-25} \mathrm{~cm}^{2}$. If $u$ is the energy density of the soft photons the number density of these photons will be $n=u / \varepsilon$ and the mean free path for photon-photon absorption is $\lambda=1 / n \sigma$.

Here I assume that the volume occupied by the optical and X-ray photons is the same as or larger than that from which the pulsed $\gamma$-rays may originate. In addition, I consider that the emission of the optical and X-ray pulses is isotropic and that their distribution in the source is also isotropic.

First I consider absorption between optical photons and high energy $\gamma$-rays, $E_{\gamma} \sim 10^{12} \mathrm{eV}$. If the Crab Nebula is located at a distance of $2 \mathrm{kpc}$ the total optical luminosity ${ }^{2}$ of the pulses is $10^{34} \mathrm{erg} \mathrm{s}^{-1}$. The amplitude of the inter- pulse is about 30 per cent of the main pulse. The total energy in the main pulse is $3 \times 10^{31} \mathrm{erg}$, corresponding to $10^{43}$ photons during the pulse. The maximum size of the object ought to be comparable with the distance travelled by light in one pulse duration, $10^{8} \mathrm{~cm}$, and the source volume should be $4 \times 10^{24} \mathrm{~cm}^{3}$. The number density of optical photons is $2.5 \times 10^{18} \mathrm{~cm}^{-3}$ and the mean free path $\lambda \sim 4 \times 10^{6} \mathrm{~cm}$

Second, I consider absorption between $\mathrm{keV} \mathrm{X}$-rays and $\gamma$-rays of energy greater than $50 \mathrm{MeV}$. Fritz et al..$^{3}$ have detected pulsed $\mathrm{X}$-rays in the photon energy region 1 to $13 \mathrm{keV}$ and found that 5 per cent of the total X-rays from the Crab Nebula were pulsed, with the pulsed component equally divided betwcen the main pulse and the interpulse. The X-ray pulsar flux is about $2 \times 10^{-9}$ erg $\mathrm{cm}^{-2} \mathrm{~s}^{-1}$ or $6.6 \times 10^{-12} \mathrm{erg} \mathrm{cm}^{-2}$ during the pulse where the pulse durations havo been taken as $3 \mathrm{~ms}$ and the repetition rate as $30 \mathrm{~Hz}$. The total energy in the X-ray pulse is $3 \times 10^{33} \mathrm{erg}$ corresponding to $\sim 2 \times 10^{42} \mathrm{keV} \mathrm{X}$-rays per pulse. The number density of these photons is $5 \times 10^{17}$ $\mathrm{cm}^{-3}$ and the mean free path $\lambda \sim 2 \times 10^{7} \mathrm{~cm}$. Recently an upper limit of $8 \times 10^{-10} \mathrm{erg} \mathrm{cm}^{-2} \mathrm{~s}^{-1}$ has been measured 8 for the pulsed X-ray flux between $30 \mathrm{keV}$ and $120 \mathrm{kcV}$ from the Crab Nebula. These results lead to a mean free path $\lambda>4 \times 10^{7} \mathrm{~cm}$.

If the optical and X-ray pulsed fluxes are joined by a straight line and the conditions mentioned are correct, then pulsed $\gamma$-rays of energy greater than about $50 \mathrm{MeV}$ will be strongly absorbed in the source.

If pulsar models ${ }^{9,10}$ where the radiation is beamed are considered, then the photons will not bo uniformly distributed throughout the source region and the absorption cannot be cstimated. There are a number of effects that introduce large uncertainties. (i) The radiation will come out at small angles and the energy thresholds and photonphoton collision frequencies will be modified. (ii) The total optical and $\mathrm{X}$-ray pulse luminosity may be either increased or decreased because we may be at the centre of the main radiation lobe or on the edge of or even in a side lobe. (iii) The radio, optical and X-ray pulses occur with the same period and pulse width but could be produced in the source at slightly different times. (iv) The volume of the source could be larger by many orders of magnitude. The upper limit on the radio and optical size of the source region is $<0.1 \mathrm{~s}$ of arc, that is, $3 \times 10^{15} \mathrm{~cm}$, comparod with the size as deduced from the pulse width, $10^{8} \mathrm{~cm}$. The radiation could come from high energy particles travelling towards the observer and the pulse width as measured could be the difference between the particle velocity and that of the radiation.

In conclusion, high energy pulsed $\gamma$-rays of energy greater than about $50 \mathrm{MeV}$ will suffer severe absorption if the optical and X-ray photons are isotropic in the source region; if beaming of the radiation occurs, as seems more probable, then the absorption, if any, cannot be estimated.

I thank Professors K. Greisen and J. Delvaille for helpful and detailed discussions.

\section{B. MCBreen*}

Clark Hall,

Cornell University,

Ithaca, New York.

Received May 19; revised September 30, 1969.

* On study leave of absence from the Department of Physics, University College, Dublin.

1 Streliu, D. H., and Reifenstein, F. C., Science, 162, 1481 (1968).

${ }^{2}$ Cocke, W. J., Disney, M. J., and Taylor, D. J., Nature, 221, 525 (1969). ${ }^{3}$ Fritz, G., Henry, R. C., Meckins, J. F., Chubb, T. A., and Friedman, H.,
Science, 164, 709 (1969).

4 Felten, J. E., Phys. Rev. Lett., 15, 1003 (1965).

${ }^{5}$ Gould, R. J., and Schreder, G., Phys, Rev. Lett., 15, 253 (1966).

' Jelley, J. V., Phys. Rev. Lett., 16, 479 (1966)

? Jelley, J. V., Nature, 211, 472 (1966).

${ }^{8}$ Hillier, R. R., Jackson, W. R., Murray, A., Redfern, R. M., and Standing, K. G., Nature, 222, 149 (1969).

${ }^{9}$ Gold, T., Nature, 218, 731 (1968).

${ }^{10}$ Gunn, J. E., and Ostriker, J. P., Phys. Rev. Lett., 22, 728 (1969). 